\title{
Identidade social de pessoas com condições raras e ausência de diagnóstico: contribuições a partir de Hall, Honneth e Jutel
}

\author{
Social identity of people with rare conditions and the lack of \\ diagnosis: contributions based on Hall, Honneth and Jutel
}

Jacqueline de Souza Gomes (https://orcid.org/0000-0002-8609-5893) ${ }^{1}$

${ }^{1}$ Instituto do Noroeste Fluminense de Educação Superior, Universidade Federal Fluminense. Av. João Jasbick s/n, Aeroporto. 28470-000 Santo Antônio de Pádua RJ Brasil. jsgomes@id.uff.br

\begin{abstract}
We acknowledge that people with rare conditions constitute a group with a specific social identity and seek to understand what the implications of this acknowledgement are either in the stigmatization or emancipation of these individuals. We base ourselves on the observation that many people who are said to have rare conditions are told that their symptoms constitute a "rare disease," without specifying which illness it might be. And, in this respect, many people with rare conditions are considered "handicapped," people with "learning difficulties," or are given many other labels that are not always socially well accepted. This article is structured around three analytical standpoints, basing ourselves on Stuart Hall, Axel Honneth and Annemarie Jutel. Initially, we discuss the construction of the social identity of people with rare conditions based on Hall's definition of the "identity crisis." We then examine the identity of people with rare conditions from the perspective of the theory of justice as recognition, especially in relation to what Honneth refers to as "reciprocal recognition." Lastly, we highlight some of the specificities of the demands for recognition of people with rare conditions - albeit without a diagnosis - basing our study on the sociology of diagnosis from the standpoint of Annemarie Jutel. Key words Rare diseases, Identity crisis, Diagnosis
\end{abstract}

Resumo Reconhecemos que pessoas com condições raras são um grupo com identidade social e buscamos compreender quais as implicações deste reconhecimento quanto à estigmatização ou à emancipação delas. Amparamo-nos na observação de que muitas das pessoas rotuladas com condições raras recebem a informação de que seus sintomas caracterizam uma "doença rara", mas não thes é especificada qual seja. E, nesta medida, muitas pessoas com condições raras tornam-se também "deficientes", pessoas com "dificuldades de aprendizagem" ou tantos outros rótulos que nem sempre são bem vistos socialmente. Este artigo está organizado a partir de três eixos de análise, fundamentando-nos em Stuart Hall, Axel Honneth e Annemarie Jutel. Primeiramente, discutimos a construção da identidade social de pessoas com condições raras a partir de uma releitura sobre a "crise de identidade" nos termos de Hall. Em seguida, examinamos a identidade das pessoas com condições raras pela ótica da teoria de justiça como reconhecimento, especialmente em função do que Honneth apresenta como "reconhecimento reciproco". Por fim, pontuamos algumas das especificidades das demandas por reconhecimento de pessoas com condições raras, mas sem diagnóstico, fundamentando-nos na sociologia do diagnóstico, a partir de uma interlocução com Annemarie Jutel. Palavras-chave Doenças raras, Crise de identidade, Diagnóstico 


\section{Introdução}

Debates sobre reconhecimento e estigmatização em saúde têm sido bastante frequentes. Contudo, pensar especificamente sobre a construção da identidade social de pessoas com condições raras englobando uma reflexão sobre as pessoas sem diagnóstico é pouco usual. A construção simbólica sobre o conceito de saúde tende a assumir perspectivas minimamente direcionadas para pessoas com condições raras, salvo análises sobre judicialização e custos para os sistemas de saúde. Para este ensaio, encampamos a saúde como direito e, desta maneira, como um conceito em permanente construção pela sociedade. É o contexto que irá determinar se nossa identidade social será oprimida ou não e, consequentemente, se teremos violados certos direitos, como $\mathrm{o}$ acesso à vida e à saúde dignas. A saúde como direito é, pois, uma conquista social que revela conflitos de interesses ínsitos à tessitura social e, nos termos de Axel Honneth ${ }^{1}$, demanda luta por reconhecimento.

Conquistar a saúde como direito exigiu (e exige) lutas de segmentos sociais muitas vezes excluídos dos debates públicos. Lutas que são exercício de cidadania e legitimação de uma sociedade que se pretende democrática. Vozes e demandas estigmatizadas pulsam, permeadas por valores intransigentes sobre quem merece ou não a titularidade de certos direitos. Ou somos unissonamente favoráveis quanto ao exercício do direito de acesso a medicamentos de alto custo por pessoas com condições raras? Pequenas transgressões em nossa forma de olhar o mundo e as pessoas podem ser necessárias a fim de ressignificarmos binômios solidificados no imaginário social (como saúde e doença, normal e patológico, belo e feio, etc).

Organizamo-nos a partir de três eixos de análise, de natureza fundamentalmente exploratória, a saber: a) ponderações sobre a complexidade dos sentidos que envolvem a identidade social de pessoas com condições raras, apoiando-nos no "sujeito pós-moderno", de Stuart Hall²; b) exame da identidade fragmentada dos sujeitos pela ótica da teoria contemporânea de justiça como reconhecimento, especialmente quanto ao "reconhecimento recíproco" nos termos propostos por Axel Honneth ${ }^{1}$ c) problematização sobre o reconhecimento de pessoas sem diagnóstico no grupo das pessoas com condições raras de saúde, a partir de interlocução com o pensamento de Annemarie Jutel sobre a sociologia do diagnóstico, a partir dos artigos "Sociology of Diagnosis: a preliminar review"3 e "Beyond the Sociology of Diagnosis"4.

Primeiramente, situamos o debate sobre a identidade social, a desconstrução da identidade estável do sujeito moderno e seguimos em direção à compreensão do sujeito pós-moderno, fragmentado. No caso das pessoas com condições raras, o escopo que as abarca é incomensurável. No rol, estão pessoas com diversas síndromes, transtornos, doenças e, ainda, pessoas sem diagnóstico clínico. Pessoas que são e estão no mundo. São medicadas, se alimentam, respiram, dormem, acordam, usam serviços públicos, etc. No entanto, por vezes, são identificadas socialmente como inferiores ou desiguais de modo a serem desrespeitadas em seus direitos mais básicos, como o direito à vida e à saúde dignas.

Este primeiro eixo de análise busca chamar atenção para a complexidade de se identificar alguém com uma condição rara, já que tal identificação é simbólica e, para além da condição individual, leva a uma série de julgamentos e condenações sociais. Se os marcadores culturais de classe, gênero, sexualidade, raça e outros eram até o séc. XX capazes de localizar o sujeito a partir de uma identidade estável, não podemos mais afirmar-nos hoje com convicção a partir destes referenciais de estabilidade. Stuart Hall ${ }^{2}$ nos coloca defronte a certezas sobre nossas identidades pessoais, chamando-nos atenção para a fragmentação da idéia que temos de nós mesmos. Este deslocamento do sujeito é, segundo o autor, fruto de uma "crise de identidade". Neste sentido, com $\mathrm{Hall}^{2}$, percebemos que a complexificação das sociedades na modernidade tardia expõe uma necessidade de se deslocar o sujeito no âmbito das próprias estruturas dinâmicas que compõem a sociedade. Consequentemente, reconhecemos também haver uma dinamicidade intrínseca ao grupo de pessoas com condições raras, representada pelo conjunto de identidades fragmentadas e passíveis de ressignificação.

No segundo eixo passamos à análise do tratamento conferido às pessoas identificadas com condições raras pela ótica da teoria de justiça como reconhecimento nos termos formulados por Axel Honneth ${ }^{1}$. Tratamento que, por vezes, fere a autoconfiança, o auto-respeito e auto-estima, gerando demanda por reconhecimento. Ressalto que não enfrentaremos neste artigo o clássico debate entre Honneth e Nancy Fraser. Tampouco aprofundaremos aspectos positivos e negativos sobre o funcionamento do Sistema Único de Saúde Brasileiro. Nosso objetivo é, a partir do conceito de "reconhecimento recípro- 
co", defender a luta por reconhecimento de pessoas sem diagnóstico no grupo das pessoas com condições raras.

Honneth $^{1}$ nos remonta a Hegel para reestruturar a idéia de "reconhecimento recíproco". Nossa identidade é construída com base no reconhecimento recíproco, que nos poderá promover autoconfiança, auto-respeito e auto-estima. Se nos ferem a autoconfiança, o auto-respeito ou a auto-estima, desdobra-se a necessidade de luta por reconhecimento. É com Honneth ${ }^{1}$ que concebemos que nossa inserção social se dá pela luta por reconhecimento, e não pela luta por autopreservação, como Hobbes e Maquiavel. Defendemos que o enquadramento no grupo das pessoas com condições raras independe de diagnóstico e de uma identidade estável. Ausência de diagnóstico não pode ser empecilho para que sujeitos sejam incorporados socialmente, inclusive para fins administrativos (como, por exemplo: reembolso, acesso a medicamentos, grupos de apoio e aposentadoria).

Por fim, nosso último eixo de análise pontua algumas das especificidades das demandas de pessoas sem diagnóstico, fundamentando-nos na sociologia do diagnóstico. Sobre a sociologia do diagnóstico, utilizaremos como referência escritos da professora Annemarie Goldstein Jutel, da Universidade de Wellington. Neste sentido, destacaremos os seguintes artigos: a) "Sociology of Diagnosis: a preliminar review", publicado em 2009, capítulo do livro "Sociology of Diagnosis", de 2011, organizado por Barbara Katz Rothman, P. J. McGann e David J. Hutson. Neste, Jutel busca estabelecer uma identidade para a sociologia do diagnóstico; e, b) "Beyond the Sociology of Diagnosis", de 2015.

Além de Jutel, vale destacar outros autores que têm refletido na esfera de uma sociologia do diagnóstico, como: Brown ${ }^{5}$; Rosemberg ${ }^{6}$; Fuat et al. ${ }^{7}$; Olafsdottir e Pescosolido ${ }^{8}$; Cambrosio et al. ${ }^{9}$; Timmermans e Haas ${ }^{10}$; Aronowitz ${ }^{11}$; Whooley ${ }^{12}$; Kokanovic et al. ${ }^{13}$.

\section{Sentidos da identidade social de pessoas com condições raras}

O Auto Retrato

No retrato que me faço

- traço a traço -

às vezes me pinto nuvem, às vezes me pinto árvore...

às vezes me pinto coisas de que nem há mais lembrança... ou coisas que não existem mas que um dia existirão... e, desta lida, em que busco - pouco a pouco minha eterna semelhança, no final, que restará? Um desenho de criança... Terminado por um louco!

Mário Quintana (2005)

Nossa existência tende a ser condicionada pela convivência social. Ao nascermos encontramos a vida organizada, herdamos heranças culturais e agregamos influências do meio onde estamos inseridos. Esse processo de socialização, dinâmico e contraditório, é intrínseco à condição humana. Como diz o poema de Mário Quinta$\mathrm{na}^{14}$, começamos a vida como desenho de criança a traduzir-nos como esperança e terminamos como loucos, à procura de luz a guiar-nos os passos desequilibrados. Neste sentido, o sentimento de pertença ao mundo social é, em regra, determinado pela nossa participação em grupos. Por vezes, nossas condições biológicas nos incluem em grupos sociais que nos levam a sofrer injustiças. E, assim, nossa auto-imagem, positiva ou negativa, vai desvelando-se como expressão do vínculo, ora afetivo, ora fleumático, que temos com certos grupos.

A identidade social expressa no reconhecimento social, por um lado, pode ser também fator de estigmatização, de outro. Estigmas que, segundo os gregos, eram marcas corporais que identificavam o status moral (escravo, criminoso ou traidor) de quem as possuíam, tornando-os inabilitados para a aceitação social ampla ${ }^{15}$. Considerando esta dupla possibilidade, entendemos o reconhecimento social como uma demanda por justiça social e a estigmatização como um fator de injustiça social.

A clássica Teoria da Identidade Social, de Henri Tajfel ${ }^{16}$, fornece subsídios iniciais para pensarmos o reconhecimento social (intra e intergrupos) de pessoas com doenças raras como instrumento de justiça social. Grosso modo, esta teoria visa explicar como as pessoas criam e definem seus lugares na sociedade, quer em termos de autoidentidade, quer em termos de heteroidentidade. Ou seja, segundo Tajfel e Turner ${ }^{17}$, uma pessoa é, em parte, constituída pelos grupos aos quais pertence. É fato que, dependendo do grupo social onde se encontre, uma pessoa poderá agir de maneiras específicas e distintas em relação a outras. Se estamos no grupo dos pais 
da escola, por exemplo, não nos comportaremos do mesmo modo que se estivermos no grupo dos jogadores de futebol.

Tajfel e Turner ${ }^{17}$ propuseram três processos mentais para a avaliação dos outros como "nós" (dentro do grupo) ou como "eles" (fora do grupo): categorização, identificação social e comparação social. A categorização é o que nos tende a identificar certas pessoas a partir de categorias sociais particulares, como, por exemplo, ao pensarmos em Bell hooks, necessariamente a categorizamos como feminista. Bell hooks é uma autora norte-americana com estudos sobre relações sociais opressivas. Seu estilo é inconfundível, sincero e, ao mesmo tempo, perturbador. Traz-nos com uma linguagem simples questionamentos complexos, especialmente sobre as opressões que sofrem as mulheres negras. Em meio a inúmeras publicações, destaque-se seu livro "Ensinando a Transgredir: a educação como prática de liberda$\mathrm{de}^{118}$. Neste, a autora busca, a partir de narrativas autobiográficas, estabelecer bases para compreendermos a educação como ato contrahegemônico, a auxiliar-nos a transgredir fronteiras e a resistir frente às estratégias de colonização do pensamento.

A identificação social nos leva a agir em conformidade com o grupo. Assim, se nos identificamos como sendo do grupo dos democratas, buscaremos agir conforme agem os demais integrantes deste grupo. Nos categorizamos dentro de um grupo, nos identificamos a ele e, na sequência, tendemos a comparar o grupo onde estamos inseridos com outros grupos externos. Assim, a comparação social é o processo de determinação do valor ou posição social de um grupo em relação a outros, como, por exemplo, quando comparativamente determinamos que médicos tenham status social diferenciado em relação aos enfermeiros. Esta comparação social pode, por vezes, levar a situações discriminatórias e de estigmatização.

E como se constrói a identidade social das pessoas com condições raras? Há multidimensionalidade e dinamicidade quanto às características individuais, o que torna o grupo de pessoas com condições raras constituído por uma identidade de caráter mutável, atravessada por variações simbólicas e conflitos de interesses. Portanto, delimitar o escopo identitário deste grupo é extremamente complexo.

Uma alternativa de delimitação, longe de resolver a querela, passa pela concepção da Organização Mundial de Saúde segundo a qual raras são as doenças cuja prevalência seja inferior a 65 casos em 100 mil habitantes. Mesmo esta não se esquiva da inexatidão quanto ao número de doenças raras existentes no mundo. Reconhece haver uma prevalência na literatura especializada de que haja entre 6 e 8 mil diferentes delas, sendo que $80 \%$ das doenças raras tendem a ser decorrência de fatores genéticos enquanto $20 \%$ tendem a resultar de causas ambientais, infecciosas, imunológicas, dentre outras. A estimativa é de que 13 milhões de brasileiros tenham alguma doença rara ${ }^{19}$.

Consideramos que um enquadramento para as pessoas com condições raras nos remete ao enfrentamento da questão da "crise de identidade", levantada por Stuart Hall (2). Passando por uma análise sobre o essencialismo cartesiano pautado na razão como elemento autodeterminante para a identidade do sujeito e pela reflexão sobre o sujeito iluminista que, não obstante ainda ter uma essência, se estabelece na relação com a sociedade, Hall (2) se ocupa em fundamentar uma idéia que se aplique à identidade do sujeito na pósmodernidade.

Ouso dizer que a "crise de identidade" a que nos chama atenção Hall (2) no início da década de 1990 relaciona-se também com o avanço do conhecimento sobre o genoma humano. Estudos relacionados ao mapeamento genético reconfiguraram a própria identidade dos sujeitos e permitiram diagnóstico precoce das causas genéticas de inúmeras patologias. Ainda assim, o que somos é, mais do que nunca, provisório, fragmentado, instável e problemático.

O sujeito pós-moderno é aquele que, segundo Hall (2), não possui uma "identidade fixa, essencial ou permanente"12. É caracterizado por uma identidade não unificada em torno de um self, mas constitui-se por identidades contraditórias e que são deslocadas com frequência. É a partir deste fundamento que afirmamos que somos todos(as) raros(as). O que nos leva a esta premissa é o fato de que somos um emaranhado de identidades possíveis. $\mathrm{O}$ fato, inclusive, de termos o gene que nos predispõe ao diabetes, não significa que seremos necessariamente diabéticos. Há fatores conjunturais e não estritamente biológicos que definirão nossas identidades.

Pensar sobre a identidade social de pessoas com condições raras é pensar sobre a identidade como campo de possibilidades, o que nos leva à nossa segunda hipótese: a de que esta identidade fragmentada demanda o reconhecimento recíproco para sua materialidade. É, nos termos de Hall (2), este duplo deslocamento do sujeito (do seu lugar social e da compreensão da uma unida- 
de de si) que nos faz demandar luta por reconhecimento intersubjetivo.

\section{Reconhecimento recíproco e identidade social de pessoas com condições raras}

Muito embora ponderações sobre justiça sejam fundamentalmente relacionadas a indivíduos, é possível também estendê-las a grupos. A justiça é, pois, uma questão de reivindicação, quer frente a outra pessoa, quer frente a instituições. Há diferentes concepções de justiça a depender de qual teoria as fundamente. Para este ensaio, utilizaremos o enfoque dado por Axel Honneth $^{1}$. Representante da Teoria Crítica, Honneth direciona-se para uma teoria cujo enfoque é a construção social da identidade (pessoal e coletiva). Há abrangentes formas de se compreender a Teoria Crítica. Compreende o pós-estruturalismo, o desconstrutivismo e tantas outras que, em regra, não se conectam à Teoria Crítica da Escola de Frankfurt, defensora de um hegelianismo de esquerda. A obra de referência de Honneth é "Luta por Reconhecimento - A gramática moral dos conflitos sociais", onde o autor privilegia não a luta pela auto-conservação ou a luta pelo poder, mas a luta por reconhecimento, nos termos propostos inicialmente por Hegel.

A questão do reconhecimento é apontada pelo jovem Hegel e reinterpretada por Honneth ${ }^{1}$. Fenomenologicamente, pela perspectiva hegeliana, passamos de uma consciência de "objetos" a uma "consciência de si" e, ainda, de uma dimensão do "conhecimento" à do "reconhecimento" dos sujeitos. Assim, os problemas da consciência não se restringiriam à relação do sujeito isolado com seus objetos. Hegel traz-nos uma perspectiva de "consciência relacional". A dialética do senhor e escravo é uma descrição de um modelo de relação entre consciências mobilizadas por uma demanda de reconhecimento. As relações intersubjetivas são relações de dominação e servidão; não são, portanto, desde seu início, relações de simetria. Neste sentido, não seria de todo inapropriado inferir que nos sentimos valorizados, em quaisquer esferas de nossas vidas, quando somos reconhecidos por terceiros. Para Hegel, o reconhecimento é o que permite-nos estabelecer uma relação ética com outros sujeitos. Nossa consciência existindo em relação a outras consciências. Mas como se estabelecerá este reconhecimento entre consciências?

Considerando que os sujeitos forjam suas identidades na relação uns com os outros, Honneth $^{1}$ afirma que os conflitos são inevitáveis. É algo utópico conceber uma sociedade onde as pessoas vivam em plena harmonia. Honneth ${ }^{1}$ vê nos conflitos não um caminho para as guerras civis, mas uma alternativa ao reconhecimento de demandas específicas, desejos e esperanças não atendidos. Nesta medida, os conflitos simbolizam algo que deve ser revisto, sinalizam algo errado no funcionamento de uma sociedade que precisa ser reavaliado. Na maioria das vezes, os conflitos sociais são induzidos não pelo egocentrismo, mas por um sentimento de que a pessoa não está suficientemente incluída, respeitada e reconhecida pela sociedade. Os conflitos são, pois, um indicativo de que há algo de equivocado quanto ao processo de reconhecimento social de uma sociedade.

E o que Honneth ${ }^{1}$ concebe por reconhecimento? O autor observa que o reconhecimento surge da idéia de que, para fazer parte de uma sociedade, precisamos ser reconhecidos. Por exemplo: só seremos livres se formos socialmente reconhecidos como livres. A luta pelo reconhecimento permeia situações diversas, desde a luta pelo reconhecimento do amor dos pais quando se é criança até a luta por estima social no interior de um grupo. Segundo Honneth ${ }^{1}$, o reconhecimento é uma construção intersubjetiva, dialógica e histórica a fim de as pessoas realizarem-se em três domínios básicos: amor, direitos e estima social. Estes seriam, portanto, os três tipos de reconhecimento:

a) de ser humano com determinadas necessidades;

b) de ser humano de status igual;

c) de ser humano com competências e habilidades que contribuem para a dinâmica da vida social.

Honneth $^{1}$ resgata a psicologia infantil de Winnicott pela qual o desenvolvimento infantil se apresenta com duas fases de dependência, a absoluta (simbiose) e a relativa. Há uma indiferenciação entre mãe e bebê na fase simbiótica. Aos poucos, o bebê vai se dando conta de que a mãe é parte do mundo e não parte dele. Na sequência, a criança passa a perceber o outro como alguém independente e que possui direitos. O amor surge exatamente quando a criança, autoconfiante, passa a reconhecer a outra pessoa como alguém independente a ela. Desde o nascimento, a criança passa por todos os estágios de reconhecimento. Luta inicialmente pelo reconhecimento do amor dos pais. Também quer ser reconhecida por suas competências e habilidades primorosas. E buscam ser reconhecidas com o mesmo status moral de outros membros da sociedade. Se há insatisfação quanto a algum destes 
âmbitos do reconhecimento, a criança, ao longo do tempo, não se sentirá incluída.

Os diversos conflitos sociais existentes são, segundo Honneth ${ }^{1}$, um desdobramento do desrespeito a algum dos domínios de reconhecimento, que atacam a identidade pessoal ou coletiva e que podem suscitar uma luta por reconhecimento. Como dissemos, os conflitos se iniciam já na infância, no âmbito familiar, e se estendem a demandas de grupos que não se sentem valorizados com mesmo status moral que outros grupos numa mesma sociedade, que reclamam uma paridade de status.

$\mathrm{Na}$ área da saúde, alguns conflitos sociais levam a lutas por reconhecimento de direitos, por exemplo. Será que o simples fato de um determinado medicamento não ser lucrativo justifica o seu custo exorbitante e a inviabilização de seu acesso a pessoas que dele necessitam para sobreviver? O mercado, nos termos do capitalismo contemporâneo, nos coloca que o acesso a saúde digna só é viável se atender a um quantitativo significativo de beneficiários. No contrafluxo, uma das lutas por reconhecimento das pessoas com condições raras envolve o acesso a saúde digna, com qualidade de vida.

Ao nos inclinarmos ao uso do termo condições raras nos é possível refletir para além das pessoas com "doenças raras" e, consequentemente, sobre as pessoas que não possuem diagnóstico de "doença", mas que, ainda assim, podem ser incluídas no grupo dos que possuem "raridade". Como dizemos acima, somos todos(as) raros(as) em alguma medida já que há variações conjunturais que irão determinar quando e quem somos. Nossa identidade tem caráter fragmentário. Pode ser que hoje não tenhamos um diagnóstico de "doença rara", mas que o tenhamos no futuro próximo. A identidade de sujeitos depende do reconhecimento intersubjetivo e o desrespeito a qualquer uma das três formas de reconhecimento apontadas por Honneth: ${ }^{1}$ implica numa luta por reconhecimento. Exemplo: desrespeitando a integridade física ou psíquica de alguém, estaremos agindo contra o amor. Se excluímos alguém de uma comunidade, estamos infringindo o direito que mencionamos no início deste ensaio.

\section{Pessoas com condições raras, mas sem diagnóstico: contribuições iniciais para o debate a partir da Sociologia do Diagnóstico}

Para introduzir o debate sobre sociologia do diagnóstico vale esclarecer ao que esta preten- de. Apesar da grande importância que assume o diagnóstico para as nossas vidas, ele nem sempre é objeto de uma área específica de conhecimento. Via de regra, seu estudo insere-se no âmbito de disciplinas como Sociologia Médica ou Sociologia da Saúde e da Doença. É a partir da década de 1970, com o texto "Diagnosis as Category and Process: The Case of Alcoholism", de Blaxter, que se passa a discutir a necessidade de se estudar o diagnóstico por uma área específica. $\mathrm{O}$ estudo de Blaxter ${ }^{20}$ sobre o alcoolismo é constantemente citado na literatura da sociologia do diagnóstico e introduz as idéias de "diagnóstico como categoria" (relaciona-se à classificação médica propriamente dita) e "diagnóstico como processo" (relaciona-se à observação sobre o contexto utilizado pelo médico para fazer a classificação diagnóstica).

Algum tempo depois, Brown ${ }^{5}$ publica "Naming and Framing: the social construction of diagnosis and illness". Neste estudo, cujo enfoque são as doenças psiquiátricas, Brown ${ }^{5}$ esboça o que seria uma sociologia do diagnóstico nos termos do que conhecemos hoje. É uma referência que nos permite confirmar a importância de um estudo sociológico do diagnóstico, especialmente por nos impulsionar a pensar uma construção social do diagnóstico e não apenas restringi-lo à literatura médica. Com Annemarie Jutel, na década de 2000, é que a sociologia do diagnóstico emergirá como campo específico. Para tanto, destaque-se o livro "Putting a Name to it: Diagnosis in contemporary society" 21 e a coletânea de artigos sob a forma de livro, "Sociology of Diagnosis"22.

Categorizar, rotular, classificar, enumerar e diagnosticar são domínios muito valorizados na tradição ocidental. Para Fuller ${ }^{23}$, não apenas importa o diagnóstico ao uso terapêutico, mas também para um "uso simbólico" $(2011,239)$. O diagnóstico influencia a ordem social e a organização da vida cotidiana. Podemos até dizer que a existência das coisas depende destas serem colocadas em alguma categoria.

Jutel e Nettleton ${ }^{24}$ sugerem-nos que o diagnóstico é uma das ferramentas mais poderosas da medicina ocidental, de natureza eminentemente classificatória. Ele demarca os limites entre o "nós e os outros" e nos instrumentaliza para ações em diversos segmentos da vida cotidiana, desde o consumo de medicamentos até a escola onde estudaremos.

No artigo "Sociology of diagnosis: a preliminary review", a autora (3): a) analisa a medicina e a relação desta com o diagnóstico; b) investiga as tensões que circundam o diagnóstico. Neste tex- 
to, ela descreve como a medicina, ao se afinar com a taxonomia biológica, passa também a explorar, coletar e categorizar os seres vivos, engajando-se na organização de um sistema classificatório que irá sustentar o que entendemos por diagnóstico. O diagnóstico é, portanto, um instrumento de classificação da medicina, fruto de um "acordo" sobre a categorização de certas condições como "doenças".

Na medicina ocidental moderna, um sistema ideal de classificação é projetado para dar conta de englobar o indispensável para se prolatar o diagnóstico. Mesmo familiares tendem a buscar este sistema de classificação que leva a um "diagnóstico incontestável” que lhes poderá, inclusive, amparar afetivamente. O diagnóstico é um "conforto", um direcionamento para a "cura" ou para o "tratamento". Ausente o diagnóstico, presente a instabilidade. Portanto, pelo diagnóstico, permitir-se-á ao médico reconfigurar a identidade do sujeito, explicando-a como algo desviante da norma. Isto significa também trazer a tona valores culturais encampados por uma sociedade sobre o que é ou não aceito como normal.

A maior parte das categorizações é feita de maneira inconsciente. Naturalizamos as classificações e temos resistência para realizarmos uma análise crítica sobre as mesmas. Trazer tais domínios à baila perpassa reconhecê-los como construções culturais. Na cultura ocidental tendemos ao reducionismo dualista, o que não é tão latente em outras culturas. Cito o exemplo das hijras indianas. A Índia reconhece um terceiro gênero, não restringindo o sujeito ao binômio masculino e feminino.

O valor conferido ao diagnóstico indica como uma determinada sociedade tende a se comportar, sinaliza as vozes privilegiadas e as silenciadas. Rótulos que se evidenciam com os diagnósticos e trazem consigo alguns dos problemas sociais "ocultados", como o das pessoas com dificuldades de aprender. Por isto, reconhecemos a existência de outras formas de se praticar a medicina e, consequentemente, diferentes abordagens para com o diagnóstico. Nos concentramos aqui, como as clássicas abordagens da sociologia do diagnósti$\mathrm{co}^{6}$, na medicina ocidental moderna.

Destaco Sarah Nettleton ${ }^{25}$, professora na Universidade de York, e seu artigo "I just want permission to be ill': towards a sociology of medically unexplained symptoms", de 2006. Neste, Nettleton ${ }^{25}$ nos traz resultados de uma pesquisa com pessoas que vivem na Inglaterra com sintomas, mas sem diagnósticos. A autora preocupase em mostrar a angústia que envolve a falta de um diagnóstico e nos faz refletir que, mesmo na inexistência de um nome para uma doença, não parece justo que alguém sofra por isto.

Desafiador é, portanto, situar o debate em função das pessoas que integram o grupo dos que não apresentam diagnóstico. Uma mãe poderá passar meses com seu filho recém-nascido internado em uma UTI neonatal, com quadros clínicos instáveis, e sair do hospital sendo direcionada para generalistas e especialistas médicos que não identificarão de imediato (ou até nunca identificarão) um diagnóstico para a criança. Casos como este, aliás, são mais comuns do que se possa imaginar. E, então, como categorizar esta criança nos termos da medicina ocidental?

A peregrinação dos pacientes em consultórios em busca de diagnóstico de condições raras é sofrida e demorada. Caroline Huyard ${ }^{26}$, no artigo What, if anything, is specific about having a rare disorder? Patients judgements on being ill and being rare, expõe que, não obstante inúmeros esforços para compreender e melhorar a vida das pessoas com doenças raras, persiste uma incerteza quanto à natureza específica destas doenças. A autora faz entrevistas semiestruturadas com 29 pacientes e 15 progenitores de crianças com doenças raras, na França. Por esta pesquisa quantitativa, a autora busca identificar: (a) até que ponto as pessoas diagnosticadas com doenças raras concebem a raridade como causa para dificuldades específicas em vários âmbitos de suas vidas ; b) como estes impactos se relacionam com outras causas além da raridade; c) como a raridade da doença pode estar relacionada a outros aspectos da vida cotidiana do paciente.

Interessante que os resultados alcançados por Huyard ${ }^{26}$ expressam que muitas situações pelas quais frequentemente passam as pessoas com condições raras não são consideradas por estas mesmas pessoas como raras e injustas. Segundo Huyard $^{26}$, os pacientes com doenças raras têm forte desejo para conhecerem e se relacionarem com outras pessoas com condições raras, o que implica na grande valia conferida às associações de pacientes e familiares. Além disto, considerando como característica intrínseca às condições raras o diagnóstico tardio, é comum uma maior preocupação com qualidade de vida em detrimento de uma busca pela cura. Indo além, a raridade implica na construção ou no estreitamento de laços de respeito e amorosidade entre aqueles que se identificam com o grupo.

À luz da sociologia do diagnóstico podemos colocar em termos sociais o debate sobre os sentidos de saúde e doença; os impactos multidi- 
mensionais do diagnóstico; as relações de poder, construção de identidades e as discriminações por trás de diagnósticos e laudos médicos; a comercialização de diagnósticos e a relação desta com a oferta de medicamentos, procedimentos e tratamentos; o autodiagnóstico e o cyberdiagnóstico; as relações entre laudo médico e inclusão nos espaços educativos; e, por fim, a ausência de diagnóstico e de categorização de doenças.

\section{Considerações finais}

Neste artigo, utilizamos como suportes as teorias da identidade social e da justiça como reconhe- cimento para defendermos o uso da expressão "condições raras" em detrimento de "doenças raras". Estamos certos de que a sociologia do diagnóstico pode muito contribuir para uma compreensão de diagnóstico que não se fixa nos limites do conceito de doença e, sendo assim, poderá nos permitir uma ressignificação de conceitos como diagnóstico, doenças, doentes e diversidade. Não há como discordar de que o diagnóstico afeta imensamente a qualidade de vida das pessoas e, inclusive, podem deformar subjetividades e conduzir à estigmatização do sujeito e de sua família. Estigmas que marcam profundamente os modos de ser de uma pessoa.

\section{Referências}

1. Honneth A. Luta por Reconhecimento: a gramática moral dos conflitos sociais. São Paulo: Editora 34; 2003.

2. Hall S. A Identidade Cultural na Pós-modernidade. $11^{\mathrm{a}}$ ed. Rio de Janeiro: DP \& A Editora; 2006.

3. Jutel AG. Sociology of Diagnosis: a preliminar review. Sociol Health Illn 2009; 31(2):278-299.

4. Jutel AG. Beyond the Sociology of Diagnosis. Sociology Compass 2015; 9/9:841-852.

5. Brown P. Naming and Framing: the social construction of diagnosis and illness. J Health Soc Behav 1995: $\left(\right.$ Spec. $\left.n^{\circ}\right): 34-52$.

6. Rosemberg C. The Tyranny of Diagnosis: specific entities and individual experience. Milbank Q 2002; 80(2):237-260.

7. Fuat A, Hungin APS, Murphy JJ. Barriers to accurate diagnosis and effective management of heart failure in primary care: qualitative study. BMJ 2003; 326(7382):196-200.

8. Olafsdottir S, Pescosolido BA. Constructing Illness: howthe public in eight Western nations respond to a clinical description of 'schizophrenia'. Soc Sci Med 1982; 73(6):929-938.

9. Cambrosio A, Keating P, Bourret P. Regulating Diagnosis in Post-Genomic Medicine: Re-Aligning Clinical Judgment? Soc Sci Med 2011; 73(6):816-824.

10. Timmermans S, Haas SA. Towards a Sociology of Disease. Sociol Health Illn 2008; 30(5):659-676.

11. Aronowitz R. When do symptoms become a disease? Ann Intern Med 2001; 134(9 Pt 2):803-808.

12. Whooley O. Diagnostic Ambivalence: Psychiatric Work arounds and the Diagnostic and Statistical Manual of Mental Disorders. Sociol Health Illn 2010; 32(3):452-469.

13. Kokanovic R, Bendelow G, Philip B. Depression: the ambivalence of diagnosis. Sociol Health Illn 2013; 35(3):377-390.

14. Carvalhal TF. Mario Quintana: poesia completa em um volume. Rio de Janeiro: Nova Aguilar; 2005.

15. Goffman E. Estigma - Notas sobre a Manipulação da Identidade Deteriorada. Rio de Janeiro: Zahar; 1978.
16. Tajfel H. Grupos Humanos y Categorías Sociales - Estudios de Psicología Social. Barcelona: Editorial Herder; 1984.

17. Tajfel H, Turner J. An integrative theory of intergroup conflict. Monterey: Brooks/Cole; 1979.

18. Hooks B. Ensinando a Transgredir: a educação como prática de liberdade. São Paulo: Martins Fontes; 2013.

19. Brasil. Ministério da Saúde (MS). Portal do Ministério da Saúde. [Online]. [acessado 2018 Nov 3]. Disponível em: http://portalms.saude.gov.br/atencao-especializada-e-hospitalar/especialidades/doencas-raras

20. Blaxter M. Diagnosis as Category and Process: The Case of Alcoholism. Soc Sci Med 1978; 12:9-17.

21. Jutel AG. Putting a Name to it: Diagnosis in contemporary society. Baltimore: The Johns Hopkins University Press; 2011.

22. McGann P, Hutson D, Rothman BK. Sociology of Diagnosis. Bingley: Emerald Group Publishing; 2011.

23. Fuller PC. Navigating professional knowledges: Lay techniques for the management of conflictual diagnosis in an AD/HD support group. In: McGann P, Hutson D, Rothman BK. Sociology of Diagnosis. Bingley: Emerald Group Publishing; 2011. p. 211-232.

24. Jutel AG, Nettleton S. Towards a sociology of diagnosis: reflections and opportunities. Soc Sci Med 2011; 73(6):793-800.

25. Nettleton S. I just want permission to be ill': towards a sociology of medically unexplained symptoms. Soc Sci Med 2006; 62(5):1167-1178.

26. Huyard C. What, if anything, is specific about having a rare disorder? Patients judgements on being ill and being rare. Health Expectations 2009; 12(4):361-370.

Artigo apresentado em 04/02/2019

Aprovado em 24/08/2019

Versão final apresentada em 26/08/2019 